Cahiers d'études italiennes

\title{
Formes, modalités et paradoxes de la séduction dans Il bastardo di Mautàna de Silvana Grasso
}

\section{Margherita Marras}

\section{(2penEdition}

\section{Journals}

Édition électronique

URL : http://journals.openedition.org/cei/815

DOI : $10.4000 /$ cei.815

ISSN : 2260-779X

Éditeur

UGA Éditions/Université Grenoble Alpes

\section{Édition imprimée}

Date de publication : 15 septembre 2006

Pagination : 69-80

ISBN : 978-2-84310-086-4

ISSN : 1770-9571

Référence électronique

Margherita Marras, «Formes, modalités et paradoxes de la séduction dans // bastardo di Mautàna de Silvana Grasso », Cahiers d'études italiennes [En ligne], 5 | 2006, mis en ligne le 15 mars 2008, consulté le 27 mars 2021. URL : http://journals.openedition.org/cei/815 ; DOI : https://doi.org/10.4000/cei.815 


\author{
FORMES, MODALITÉS ET PARADOXES \\ DE LA SÉDUCTION \\ DANS IL BASTARDO DI MAUTÀNA DE SILVANA GRASSO
}

\author{
Margherita Marras \\ Université d'Avignon
}

Silvana Grasso [1951] a puisé dans l'histoire, la société, l'anthropologie, et dans les topoi littéraires de son île, la Sicile, les matériaux de son monde romanesque ${ }^{1}$. Mais tout en édifiant un pont avec la tradition littéraire sicilienne, elle a cherché et trouvé le moyen de s'en détacher et d'affirmer son originalité: son écriture poussée à l'extrême, démystifiante, hybride, provocatrice et inquisitoriale. Silvana Grasso entre aussi, à juste titre, dans le "cercle» des romancières italiennes (Maria Rosa Cutrufelli, Carmen Covito, Francesca Mazzucato) qui, dans les années 90, contribuent au brouillage des codes et au bouleversement des modèles, des formes, des styles considérés comme typiquement féminins. Le dépassement d'une littérature marquée par l'appartenance biologique se renforce par une représentation de la séduction, l'un des thèmes qui réunit ces écrivaines ${ }^{2}$, réalisée sous le signe de la diversité: une confirmation supplémentaire de l'absence de modèles communs (au féminin) mais qui nous renvoie aussi au concept même de séduction dont les frontières restent multiples. Cette étude examinera les formes et les modes de la séduction dans Il bastardo di Mautàna, certainement le roman le plus transgressif de Silvana Grasso, le plus excessif et le plus imparfait, mais aussi celui qui illustre le mieux l'originalité de cette écrivaine ${ }^{3}$. L'analyse portera sur les personnages féminins

1. Nebbie di Ddraunara (1993) suivi de Il bastardo di Mautàna (1994), Ninna nanna del lupo (1995), L'albero di Giuda (1997) et La pupa di zucchero (2001).

2. Cutrufelli M. R. dans La Briganta (1990), Covito C. dans La bruttina stagionata (1992) et Mazzucato F., entre autres, dans Amore a Marsiglia (1999).

3. Grasso S., Il bastardo di Mautàna, Turin, Einaudi, 1994. Toute citation tirée de Il bastardo di Mautàna comportera le numéro de page correspondant dans le corps du texte et entre parenthèses. 
auxquels la romancière a confié le pouvoir de séduire et sur les contraintes de la séduction dont les mécanismes et les stratégies s'inscrivent dans une Sicile romanesque, provinciale et archaïque.

Il bastardo di Mautàna est structuré en cinq parties rigoureusement distinctes au niveau temporel (Mautàna 25 giugno 1921, Terranova 26 giugno 1921, Terranova 27 giugno 1921, Terranova luglio 1923, Terranova 1948), qui toutefois ne répondent pas à la réelle extension des faits racontés. Cette limitation temporelle représente en fait l'espace pour une évocation du passé (englobant toute la première moitié du XXe siècle), où l'écriture semble tendre, plus qu'à la narration, à une description se formalisant dans une répétition cyclique de gestes, comportements, faits, actions qui sont à l'origine d'une redondance autant linguistique que conceptuelle. Le lieu de l'intrigue se situe entre la campagne de Mautàna (propriété de la famille Verderame) et la ville de Terranova: un double espace, intérieur à l'île, dont Silvana Grasso ne cherche pas à mettre en valeur la diversité mais au contraire l'homogénéité, appelée à confirmer le concept de fermeture du monde insulaire avec ses obstacles extérieurs, ses inhibitions et ses paradoxes internes. C'est dans ces contextes que la séduction prend forme, une séduction qui se joue sur plusieurs fronts (la soumission et la révolte, le désir mortifié et la liberté des instincts) et sur l'opposition constante entre femmes dominées et femmes dominantes. Parmi les dominées ${ }^{4}$, il y a les paysannes des «masserie» de Mautàna, dont le rôle devient expression d'une servilité sociale qui se manifeste dans leur assujettissement au désir des hommes-patrons et dans leur acceptation passive, voire fataliste, des formes ritualisées de l'exploitation sexuelle:

Lupo pensava a Mariannina che certo s'era sottomessa a don Giachino pensando ch'era giusto, che coi padroni non si discute mai - e che mai altro poteva fare ? non aveva fatto così sua madre ? e la madre di sua madre ? che pure erano state brave e timorate di Dio. Ed erano morte col conforto dei sacramenti. (Il bastardo, p. 55)

Dans la représentation de Silvana Grasso, il existe une libido «socialement sexuée " " selon la définition de Bourdieu, où la "libido dominantis" (désir du dominant) se réalise à travers une logique d'asservissement sexuel, issue du "droit» à la possession, qui rabaisse les paysannes à des objets de plaisir et les condamne à la perte de leur identité: «Dieci cento o mille, le contadine delle masserie Lupo sono tutte uguali... uguali ti

4. Parmi les femmes dominées, il y a aussi les épouses des propriétaires des terres de Mautàna dont la présence dans le roman est toutefois assez marginale. Il s'agit de femmes enfermées dans un art de vivre au féminin sous le signe de la plus totale vacuité.

5. Bourdieu P., La domination masculine, Paris, Seuil, 1998, p. 64. 
dico...» (p. 61). Impossible de faire l'impasse sur l'un des éléments essentiels de cette représentation, la roba, «l'un des sentiments qui conditionne le plus la vie sicilienne " ${ }^{6}$, dont I Malavoglia de Verga ont dévoilé «le côté objet et obscène", et qui a été célébrée aux suprêmes niveaux "du Pirandello de l'Esclusa et du Sciascia de $A$ ciascuno il suo " ${ }^{7}$. Comme chez le don Gesualdo de Verga, le rapport entre personne et roba se traduit dans Il bastardo di Mautàna par une perte de conscience du sentiment et donc par son inévitable absence. Avec don Giachino, la signification économique de la roba s'étend inexorablement à la femme qui, appelée à confirmer la notion de propriété, devient l'instrument de l'affirmation de sa puissance et de la légitimation de sa violence érotique. Au même titre que la roba, elle est assujettie au droit héréditaire: ainsi, don Giachino promet à son fils naturel que, une fois devenu évêque, il pourra posséder "poderi e pecore e... le femmine delle masserie» (p.60), "quante ne vuoi... intesi ?... intesi Lupo...?» (p.45).

L'avilissement des femmes continue et se renforce à travers un procédé de sexualisation animale - digne d'un manuel d'éthologie - qui, pour les paysannes de Mautàna, se construit autour des perceptions et des descriptions fournies par don Giachino: elles sont remplies de poux qu'elles «scrafazzano con l'unghia» (p.61), soumises comme les chèvres (p.60) et muettes «dopo la monta» (p.61) comme "vitella che si stacca dal toro" (p.61); ce sont des mercenaires du sexe ou bien des "cagne in calore» (p. 45) qui offrent leur services pour « una pezza di formaggio pecorino e ti stanno addosso come zecche...» (p. 61). À cette mortification de la féminité n'échappe pas non plus l'une des femmes dominantes ${ }^{8}$ de Mautàna, Stinca, qui dénature toute éthique dans la pratique d'une séduction active. Sa caractérisation animale marque la nature de sa séduction et montre les liens qu'elle établit avec les hommes. Elle est associée aux bêtes errantes ("gatta randagia», p. 118) et indomptables (viso fiero di cavalla

6. Sciascia L., Pirandello e la Sicilia, Caltanissetta, Sciascia, 1961, p. 24.

7. Onofri M., Storia di Sciascia, Bari-Rome, Laterza, 2004, p. 131

8. L'autre femme dominante de Mautàna est Canaria, la guérisseuse, dont la figure de «maiara » renforce la particularité et les contradictions du monde de Mautàna: elle soigne avec ses herbes mais sa tâche principale est liée à l'avortement qu'elle pratique chez les paysannes, même à grossesse avancée, «che pure se la chiamavano per abortire, si facevano il segno della croce. Tre volte sul petto, perché gli ricordava il peccato " (p. 16). Les actes sexuels ne sont pas subis par la Canaria mais, souvent, calculés et en rapport avec son métier: «Poi - lo faceva apposta - mentre con rapidi gesti delle mani gli conciava la caviglia gonfia e la strofinava con sugna di capra, non si curava affatto di rimettere ordine tra i seni in fuga dal reggipetto così che il picciotto, addietro al dondolio del suo petto, avesse meno pensieri dei dolori della caviglia. E lei, se non c'erano storie si picciava prima» (p. 15). 
che ignora la sella», p.35); ses stratégies séductrices d'animal rapace ("occhio sparviero», p. 121, «sparviero assassino», p. 124) et ses pratiques sexuelles sont comparées à la perversion et à la monstruosité d'une créature imaginaire et anthropophage tel que le "Lupomannaro» (p. 145). Il s'agit ici d'une exagération, presque d'une caricature: on assiste à une sorte de dégradation de l'être humain mêlant le burlesque et le tragique, qui acquièrent une fonction narrative primordiale. Corporéité et corporalité, soumises et effacées, ou agressives et menaçantes, sont aussi à la base des choix, des gestes et des destins individuels. Figure anomale dans sa communauté, Stinca construit son comportement autour de la perception d'une corporalité menacée et à la merci de préjugés, de valeurs et de structures hiérarchiques figées. C'est ainsi que, pour ce personnage, la séduction devient un antidote à la suprématie des hommes et un moyen de contrecarrer leur violence potentielle: elle se sert de son corps, émanation d'une "féminité sauvage" et extension de sa révolte intérieure, pour déjouer les «lois» communautaires et se dresser contre l'ordre inégalitaire de sexes. Mais pour atteindre son but, Stinca doit se voir elle-même en homme, se transformer en animal prédateur pour s'emparer de sa "proie" et mener le jeu sexuel. Elle endosse ainsi la rudesse d'une «nature» masculine dont témoigne l'odeur de son sang menstruel «forte, maschio, intenso » (p. 120), corollaire à l'affirmation de sa sexualité:

Non lo fermava Stinca il suo sangue con il panno in quattro, come le contadine di Mautàna che ne avevano vergogna e li schiarivano a bagno con la cenere, di notte, i teli lordi, accorte a non farsi scoprire dagli uomini di casa. Sempre lì a nascondere ogni traccia delle loro mestruazioni, che sentivano come un evento sporco, tanto che, per dire che non avevano le mestruazioni, dicevano "pulita sono ». (p. 120)

Par cette désobéissance d'ordre moral et esthétique, entraînant un comportement qui manque à la décence, elle impose au regard d'autrui les signes de sa sexualité, rempart de son émancipation, ce qui lui permet de s'éloigner des modèles féminins établis. En laissant couler librement son sang, elle conforte la cohérence de sa révolte extrême ; elle se place au-dessus de toute contrainte sociale qui puisse dévoiler la vulnérabilité des femmes en les obligeant à des pratiques normées et en les reliant aux habitudes d'une existence soumise.

Les principes éthiques d'un art de vivre au féminin sont eux aussi constamment transgressés dans les "rites sexuels et séducteurs " de Stinca visant l'anéantissement de l'homme. Ces rites sont à l'origine d'un renversement catégorique de la "logique de la conquête » masculine mentionnée par Pierre Bourdieu, et de ses habituelles «formes de domination, d'appropriation, de "possession" ". Mais pour aboutir à ce résultat, le per- 
sonnage doit mener un combat contre tous ceux qui (et tout ce qui) entravent son chemin. Elle s'attaque ainsi à l'Église et au clergé, responsables à Mautàna d'une division draconienne des rôles légitimant la domination masculine. Pour s'affranchir de cette emprise, Stinca s'oppose à tous les préceptes chrétiens et viole la sacralité des espaces ecclésiastiques (symboliques et matériels) ; son premier rapport sexuel a lieu dans une église, aboutissement de stratégies sordides commencées pendant la liturgie :

Stinca aveva dieci anni e ne era certa che il canonico con l'occhio sudicio la guardava in modo strano [...]. Così, forse in principio per vedere se il vecchio seguitava o forse perché a Mautàna non succedeva mai niente [...] cominciò a schiuderle le cosce, pian piano, con maestria [...] in prima fila davanti all'altare, assicurandosi che ciuffi di pelo rosso venissero fuori, con quel che si vede e non si vede, dalla radura dell'inguine. Ciuffi che lei ripassava con la sugna di porco per renderli più scuri e lucenti, e vedere poi che succedeva con la curiosità maligna più dei bambini che dei furfanti [...]. Poi finita la funzione, con la scusa delle ostie, si presentò al canonico che non se lo fece dire due volte, e grasso com'era ancora coi paramenti addosso prese a rovistarle il candido fiore dei seni, l'acerba corolla dell'inguine vergine. «Canonico, dov'è lo Spirito Santo ? " gli chiedeva Stinca già a terra dietro l'altare "Canonico, dov'è lo Spirito Santo ?" Qua è... o... qua forse qua...? e indicava con l'indice i capezzoli duri come olive o la tenera sorgente del pube [...] Infine Stinca baciava il Cristo d'oro sul petto sudato del canonico e s'arrampicava, col fazzoletto pieno d'ostie benedette, e il ventre pieno degli umori del Canonico al Pianoro delle stelle, nella notte muta di Mautàna. (p. 37-38)

On retrouve dans ce passage le ton des parodies sacrées du Moyen Age où la connotation érotique ou sexuelle se superpose au territoire liturgique ${ }^{10}$. Chez Silvana Grasso, cette rencontre se matérialise par un balancement continuel entre le sacré profané et le profane sacralisé, mais aussi par l'identification biblique de Stinca avec la femme tentatrice. Une femme au pouvoir diabolique, qui, toute sa vie, affirme sa séduction à travers une sexualité violente et attractive, qui n'accepte ni refus ni obstacle : séduction immorale et coupable, qui se concentre dans les modalités d'une conquête visant à apprivoiser, captiver, jouer sur la faiblesse des hommes notamment ceux qui représentent l'autorité craignant Dieu - et les faire tomber dans l'erreur. La tentation de voir dans cette représentation une satire anticléricale est forte: elle trouve confirmation dans l'attitude double et équivoque des hommes d'Eglise, dont la dévotion et la luxure soulignent l'incohérence de l'éthique chrétienne. Le support indispensable à cette satire est le personnage de Stinca dont le comportement nous

9. Bourdieu P., La domination masculine, op.cit., p. 26.

10. Pour cette inspiration parodique voir, entre autres, Libro del buen amor de Juan Ruiz. 
ramène, encore une fois, au Moyen Age et au rôle joué par la "serrana ", femme aux approches séductrices, acharnées et impitoyables, qui affiche ouvertement la charge érotique de son corps dans le but de plier l'homme à ses incessantes prétentions sexuelles et de l'obliger à se soumettre à sa volonté :

Era dell'Ordine dei Capuccini, poco più di un ragazzo [...]. «Ci sta... certo che ci sta... come gli altri ci sta... porco comme gli altri [...]. Sotto la tonaca è fatto di carne... di carne... e viene qui a fare il santo... le mani giunte... la croce al petto... ». Più il giovane predicatore pareva serio e pregava e curava i cristiani di Mautàna, più Stinca procurava d'avvicinarlo, e tentarlo e metterlo in croce, a suo modo, s'intende. Voleva averlo a tutti i costi, ne voleva provare il fermento del sesso intatto, che solo nel pallido lino del letto, di notte e involontariamente, si abbandonava a schizzi improvvisi, trasparenti come rugiada. (p. 40-41)

Cette charge négative caractérisant la séduction apparaît absolument conforme au "vocabulaire ecclésiastique ", normée et modelée sur lui, et plus précisément sur son acception originaire de "corruption ", "terme emprunté au latin classique seductio "action de mettre à part" ". La séduction équivaut à "tromperie dans laquelle on fait tomber quelqu'un en erreur $"{ }^{11}$, et au Moyen Age (mais encore à l'époque classique) elle devient "œuvre du diable et figure du mal $»^{12}$. Stinca est «diavola» (p.26), «diavùla » (p.40), "occhiodiciàula » (p.79), «indemoniata » (p.135), et elle entretient un rapport irrévérencieux et démystifiant avec la morale familialiste, l'éthique féminine et les coercitions de nature, encore une fois, biblique. Toute l'histoire de sa vie, d'ailleurs, se déroule autour de cette désacralisation et de la déformation des prescriptions évangéliques. Ainsi, la relation de Stinca avec Tano Verderame (marié avec Leonora) remet en cause les liens sacrés du mariage. La procréation féminine se dénaturalise par la volonté nihiliste de ce personnage qui désagrège autant les valeurs chrétiennes que les lois naturelles ; sa grossesse n'est pas marquée par des signes corporels : "Non si vedeva affatto che era incinta, nemmeno una sporgenza sul ventre, niente di niente" (p.122). Stinca oppose sa désobéissance à la malédiction biblique qui condamne la femme aux douleurs de l'accouchement, en mettant au monde son enfant en silence : «Nell'aria non un grido, non un lamento, non uno spasimo » (p. 123). Le concept de rédemption et de purification liée à la naissance,

11. Dauphin C. et Farge A., "Cerner le sens des mots ", Séduction et société, Paris, Seuil, 2001, p. 8 .

12. Klapisch-Zuber C., "Les postiches de la séduction et la métaphore de la statue peinte ", Séduction et sociétés, Dauphin C. et Farge A. éd., op. cit., p. 29. 
conditionnement culturel présent dans l'Occident, est contredit par son mépris et sa violence animale :

Lo guardò, Janìa, era come un bozzolo di lombrico pieno di bava. Un pezzo di carne rossa da macello, la coscia d'un agnello o i rognoni di un toro. Coi denti, con uno strappo netto, serrò quel budello che la legava al bambino che lei aveva appena partorito. (p. 124)

Et c'est toujours par rapport à cet affrontement qu'on peut lire l'ignominie de son geste contre nature suivant l'accouchement : l'abandon et l'exposition de son enfant " ai rapaci della notte che lo finissero a beccate e ne spolpassero il cervello» (p. 124).

La logique diabolique et profanatrice de Stinca n'est pas, toutefois, un cas isolé dans Il bastardo di Mautàna : elle imprègne le roman et est placée au coeur d'une tension interférant - dans un mouvement double et inverse - avec le contexte, les personnages et leurs comportements. C'est par ces moyens que se formalise la représentation, jusqu'à l'extrême, de l'île sicilienne comme "oxymoron géographique et anthropologique ${ }^{13}$. Les termes de cet oxymoron gravitent souvent autour d'épithètes péjoratifs, d'analogies impertinentes entre le vocabulaire ecclésiastique (la crucifixion, la passion du Christ, la prière) et les nombreuses connotations sexuelles qui deviennent métaphore du triomphe de la chair et de la corporalité. Ainsi, les paysannes de Mautàna sont «nude come Gesù alla Croce e una tempesta d'umori tra il pelo rizzuto dell'inguine" (p.61) après leurs rapports sexuels. Et, contrairement à Stinca, leur grossesse est à l'origine d'une dissonance monstrueuse - autant visuelle ${ }^{14}$ qu'olfactive $^{15}$ : «Una caverna di grasse budella mosse di nuove seme di vita [...] ventre pieno, costipato di carni come budello di maiale per le salsicce della festa per la Madonna della Speranza a Terranova » (p. 55). La nef de l'église est "lunga come il budello per il sanguinaccio " (p.71) et sa coupole marquée d' "escrementi nigrosanguinelli come amarene » (p. 97); dans son bordel, «Mimina la santa " (p. 105) reçoit ses clients avec «il Rosario in mano» (p.105). Et nombreux sont encore les passages où, entre autres, la prostitution et l'homosexualité apparaissent comme inséparables des hommes et des espaces religieux.

13. Bufalino G., Cere perse, Opere 1981-1988, in M. Corti M. et F. Caputo (édit.), Milan, Bompiani, 2001, p. 859.

14. «Una pancia grande, mostruosa, anzi pareva che, eccezion fatta per la testa, null'altro avesse che pancia pancia pancia e pancia ancora " (p. 54).

15. "Puzzava d'aglio fritto e broccoli pure se la veste per il battesimo del figlio era quella della festa, ma le saliva sui fianchi, ingrassati per la gravidanza ", (p. 55). 
La combinaison de ces éléments est à l'origine d'une transposition sacrilège où semble évidente l'intention parodique de Silvana Grasso. Elle fonde son texte sur ces associations discordantes et établit une connexion logique entre le développement thématique et la dimension subversive du monde représenté. Le locus - aux contours rudes et incertains - ainsi créé amplifie le drame des personnages et justifie leur soumission ou leur révolte.

Mais on ne peut pas saisir l'importance et les raisons de ces oppositions incessantes, parfois harcelantes, sans prendre en compte la Sicile, et plus précisément sa littérature narrative et les nombreux essais qui, à maintes reprises, ont évoqué la position du Sicilien par rapport à la religion. Avec des nuances et des registres bien moins affichés et moins pressants que ceux de Silvana Grasso, Leonardo Sciascia raconte, dans La corda pazza ${ }^{16}$, une religion unissant dévotion et jurons, et Gesualdo Bufalino, dans $I l$ malpensante, affirme ce même concept ( "J'ai blasphémé longuement cette nuit, les mains jointes en prière, dans le noir $»^{17}$ ) et il évoque la mixité entre religion et amour profane en comparant "la première fois au bordel » à une «joyeuse confirmation » et à la prise des " ordres d'un sacerdoce profane " (Il malpensante, p. 1054). De son côté, Dominique Fernandez fait remarquer que la vie sentimentale des Siciliens est scindée en deux : une part "conventionnelle » et l'autre "active». La première correspond à l'amour conjugal, "ressortit au devoir, à la loi, à la religion ", tandis que la seconde appartient au domaine de la liberté et de la vraie vie érotique qui «commence dans la chambre de passe ${ }^{18}$.

Fortement récurrente dans l'œuvre de Silvana Grasso, cette dualité est aussi au coeur de la séduction. Stinca utilise la séduction comme moyen de défense, mais la perversion des mécanismes qu'elle met en place transforme cette séduction en soumission volontaire (choisir avant d'être choisie), de même d'ailleurs que les buts de ses stratégies sordides - correspondant à l'anéantissement de l'autre - entraînent inexorablement son autodestruction. Ayant choisi la séduction comme moyen d'affirmer sa transgression, Stinca, par la violation de tout pacte social, renonce d'emblée à l'amour. Elle n'en connaît que les poisons et les saveurs

16. Voir Sciascia L., La corda pazza, Turin, Einaudi, 1970, p. 193.

17. Bufalino G., Il malpensante, Opere 1981-1988, M. Corti et F. Caputo (édit.), op.cit., p. 1108.

18. Fernandez D., Mère Méditerranée, Paris, Grasset, 1965, p. 210. 
toxiques, elle sacrifie et mortifie son désir en donnant son corps à des hommes rebutants ${ }^{19}$ et à tous ceux qui se prennent dans ses filets. Par ses choix, Stinca se condamne à la défaite. Sa vie ne peut, en effet, se dérouler qu'entre une liberté absurde et un esclavage grotesque où sa séduction transgressive, entre calcul et folie, devient le signe d'une victoire éphémère. Comme dans un jeu de réfractions et d'équivalences malheureuses, ses victimes se transforment en bourreaux, et tous ses efforts aboutissent à la vacuité la plus totale. Les mécanismes de la séduction de Stinca, à l'origine de dévastations multiples, coïncident ainsi avec une éducation sentimentale à la mort qui se réalise, jour après jour, par un processus de décomposition et de consomption de la vie. Mais si toute tentative de révolte tombe dans le vide, c'est aussi parce que, dans le monde de Mautàna, aucune dialectique n'est possible, car aucun acte de rébellion ne peut desserrer l'étreinte de l'île-prison :

Ancora dopo trent'anni o più, il rintocco della campana del Carmine, le entrava nel petto, le squarciava i timpani come i diti, grossi e sudati del canonico, le avevano squarciato, da bambina, l'inguine intatto, i seni duri come nespole sul ramo. (p.39)

Dans son exhibition d'histrion, Stinca met en place les paroxysmes de la séduction et ses rituels animaux, devenant héroïne "pirandellienne ", condamnée à subir elle-même et à s'affubler de nombreux masques, tous dignes de foi et tous faux. Son parcours existentiel «du ventre maternel à son cercueil », selon la définition de la vie insulaire donnée par Gesualdo Bufalino, a été une histoire de "compartiments étanches, de capsules ", une sorte de navigation "dans un système d'écluses $"^{20}$, emblème d'un monde insulaire sous-entendant une ségrégation de l'être humain ("de la province, de la famille, de la chambre et de son propre cœur $\left.»^{21}\right)$. Silvana Grasso a fait de Stinca une "mutinée » contre la vie, et le personnage qui incarne le mieux le lien ancestral entre Eros et Thanatos : une représentation habituelle dans la littérature sicilienne, proche de la conception que les Siciliens ont (ou ont eu) de la femme. À ce propos, Leonardo Sciascia affirmait dans Pirandello e la Sicilia que «la femme, dans le pur événement érotique nous emporte vers une contemplation de la mort $»^{22}$.

19. Entre autres, le prêtre de Bagheria «grasso, aveva un occhio che sparava in fuori, grande umidiccio con poche ciglia e la palpebra inferiore rivoltata " (p.37).

20. Bufalino G., Il malpensante, op. cit., p. 1060.

21. "L'insularità [...] non è una segregazione solo geografica, ma se ne porta dietro altre: della provincia, della famiglia, della stanza, del proprio cuore ", Bufalino G., La luce e il lutto, ibid., p. 1141.

22. Sciascia L., Pirandello e la Sicilia, op. cit., p. 25. 
Cette connexion femme-mort est une note constante chez Silvana Grasso. Elle se manifeste aussi bien à travers un langage codé que dans la destinée funeste des hommes de l'entourage de Stinca.

L'emprise de Stinca sur le sexe masculin est comparée à "la peronospera che s'attacca ai vitigni e li secca» (p. 134), et le flux de son sang menstruel, emblème de sa rébellion et de son rôle de dominante, donne lieu à une représentation qui s'aligne sur le paradigme de la destruction : "Sangue che le allagava le cosce nude immacolate, giù a precipizio dall'utero aperto come la grandine d'agosto sul chicco rigonfio dell'uva » (p. 120).

Parmi ses victimes, le jeune prédicateur qui se suicide après avoir cédé aux tentations :

Lo trovarono al carrubo, di mattina presto, con la corda al collo di seta, gli occhi immoti al cielo tra confuso sciame d'api. Pendeva dal carrubo, il predicatore, quasi in pace ora che il peccato di esserci stato con quella creatura [...] si strozzava tra i lacci attorno al suo collo. (p.41)

Tano Verderame subit pendant trente ans la séduction irrésistible de Stinca. D'où une dépendance morbide et viscérale qui met sa vie en danger et dont la seule échappatoire possible est la mort, propos qui devient un refrain obsédant dans le roman ${ }^{23}:$ "L'unica cosa che Tano sapeva con certezza era "m'ammazza una volta o l'altra m'ammazza o l'ammazzo io" " (p. 118).

Les notes funèbres accompagnent aussi la théâtralité de la vie de Stinca. Ses masques tombent avec son suicide, dernier défi aux préceptes chrétiens mais surtout prise de conscience de son échec et choix extrême de purification et d'expiation - confirmé par le lieu (le caroubier où s'était pendu le prédicateur ${ }^{24}$ ) et par le moment choisi (le jour de la mort de $\left.T^{T a n o}{ }^{25}\right)$ - nécessaire pour atteindre, enfin, la liberté : «Lei [...] pendeva nell'aria libera come la passera tra le forre dell'Accia, leggera come le tamerici della palude che hanno la foglia verde e pulita pure se di fango si nutre la radice " (p. 169).

Ce geste tragique résume la vie de Stinca, véritable épigone décadent où se mêlent chair, diable et mort. Mais son histoire est aussi celle de tous les personnages qui, dans Il bastardo di Mautàna, dans la révolte autant que dans la soumission, sont condamnés à vivre prisonniers de contraintes

23. Voir p. 62, 69,108, 112, 114, 118, 122, 124 et 131.

24. "S'era ammazzata Stinca al carrubo, col cingolo del predicatore di Città che al carrubo aveva lasciato vita e peccato " (p. 169).

25. Stinca « da sé s'era ammazzata ora che non più Tano poteva farlo » (p. 19). 
obscures, dans la violence des schémas élémentaires d'un contexte répugnant. Silvana Grasso raconte les moindres aspects de ce monde en décomposition avec son écriture particulière et artificieuse, codée et allusive, où l'amalgame entre oxymorons, métaphores et analogies n'est pas toujours réussi. Son lexique est modelé sur des termes d'origines différentes (précieuse, archaïque, dialectale), ses phrases se construisent sur de fréquents mélanges linguistiques. Au registre soutenu succède un autre registre aux tons démystifiants, à l'origine d'exagérations fastidieuses, redondantes et souvent harcelantes. La représentation de la séduction et de la sexualité est confiée à une parole dont le retentissement s'amplifie dans de nombreuses associations qui se focalisent - après les symboles sacrés - sur la matière corporelle et physiologique et sur l'espace naturel.

Ces associations se manifestent au travers de confrontations abjectes entre liquides humoraux (sang, sperme, sueur, urine), viscères, appareil génital, phénomènes atmosphériques, monde végétal et "produits dérivés " : ainsi, la soumission à don Giachino des "serve delle masserie » (p. 61) aux «natiche dolci come i meloni della Piana " (p. 45) ou «spinose come zucche della Piana " (p.61) qui reçoivent le sperme ("getto giallo, colore della semola ", p. 61) de leur maître.

Le même procédé est utilisé pour agresser les symboles de la virilité. En témoignent les images horrifiantes, débordantes de violence, de fellations vampiriques :

Stinca, nella trappola delle labbra, lo serrava il nervo grande di Tano tra l'aguzzo puntiglione dei suoi denti d'avorio. [...] Inerme e pavido era Tano a fronte di quei dentini che or lo ferivano or l'animavano or l'ammazzavano come timido parto d'anguilla che cede la vita alla furia lucente dell'amo. (p. 145)

En témoigne également la putrescence dégoûtante d'un sexe rongé par la maladie, comme la hernie ("sozzo budello», p. 72) de don Giachino qui, lors de ses rapports avec les femmes des masserie, «veniva giù. Precipitava» (p.72) :

Un'ernia grassa sconsacrata grande come melone marcito sul campo gli cadeva a metà coscia, prima lenta come marmellata di mela cotogna, poi, una volta fuori dal testicolo, si lasciava andare a precipizio come grandine sulla vigna. Nel testicolo a manca i visceri grassi dell'addome s'erano fatti un varco, e vi calavano molli come ricotta, quella col siero, sì che il muscolo cedeva, quale muro senza malta alla furia del muschio. (p.72)

La symbiose des différents éléments met en scène la triade mort/femme/espace. La mort de don Giachino en est un exemple : 
Si chiusero per sempre gli occhi di don Giachino al mestruo raggio del sole che incendiava la Rocca, quando se ne stava sulla stuoia di capra accanto alla Canaria, e alle sue cosce lunghe come erano, lunghi, i tramonti sull'Accia. (p. 92-93)

Par son écriture, Silvana Grasso établit des liens significatifs entre l'être humain et les éléments naturels et/ou animaux, corollaires les uns des autres, qui se renforcent dans une sorte de contamination réciproque, dans une fusion cyclique sous le signe de la destruction et de la régénération : l'avorteuse après avoir vidé les paysannes «del misero fardello di carni [...] e [de]i germogli di vita " (p. 17), " con quello che ne tirava fuori, vi concimava la vigna » (p.73). Ou encore les hommes «deboli di polmoni » (p. 82) et les femmes, "spolpate di parto recente» (p. 82), vont à l'abattoir communal pour boire des litres de "sangue rosso come di vitello scannato [...] caldo caldo, con l'odore di stalla e fieno" (p. 82).

À cette fusion cyclique n'échappe pas non plus la séduction. Dans Il bastardo di Mautàna, celle-ci se glisse et envahit tous les espaces de Mautàna et de Terranova. Elle est l'emblème de ces mondes couleur de ténèbre, évoluant dans un éternel présent, comme en témoigne la destinée immobile d'êtres humains qui ne sont que des figurants dont les vies, dans une mise en scène infinie, se poursuivent et se reflètent.

Il bastardo di Mautàna est un "pastiche ", le résultat de nombreuses alliances entre traditions narratives différentes : d'où la difficulté d'un classement précis dans la littérature contemporaine. Le seul point indiscutable de l'écriture de Silvana Grasso reste son rapport avec la littérature sicilienne (de Verga a Bufalino en passant par Pirandello). Tout en étant soumis à une narration transgressive, ses paramètres et ses thématiques se manifestent comme des constantes, révélatrices d'un fort lien "héréditaire " avec la tradition autochtone, à laquelle peu d'écrivains siciliens ont échappé (ou échappent). La Sicile est le cœur de son inspiration créatrice, un point de départ pour représenter la crise des structures sociales, les aliénations de personnages à la merci d'une île anthropophage qui les contraint à se soumettre à la malédiction éternelle de l'immuabilité. 